\title{
Diagnostic challenges in an atypical chest pain, Tietze's syndrome: a case report in Northeast of Iran
}

Mohammad Reza Taghavi1 $^{1 D}$, Samaneh Mollazadeh $^{2 *(D)}$, Mina Sadat Mohajerzadeh Heydari ${ }^{1}$ (D)

1. Faculty of Medicine, North Khorasan University of Medical Sciences, Bojnurd, Iran.

2. Natural Products and Medicinal Plants Research Center, North Khorasan University of Medical Sciences, Bojnurd, Iran

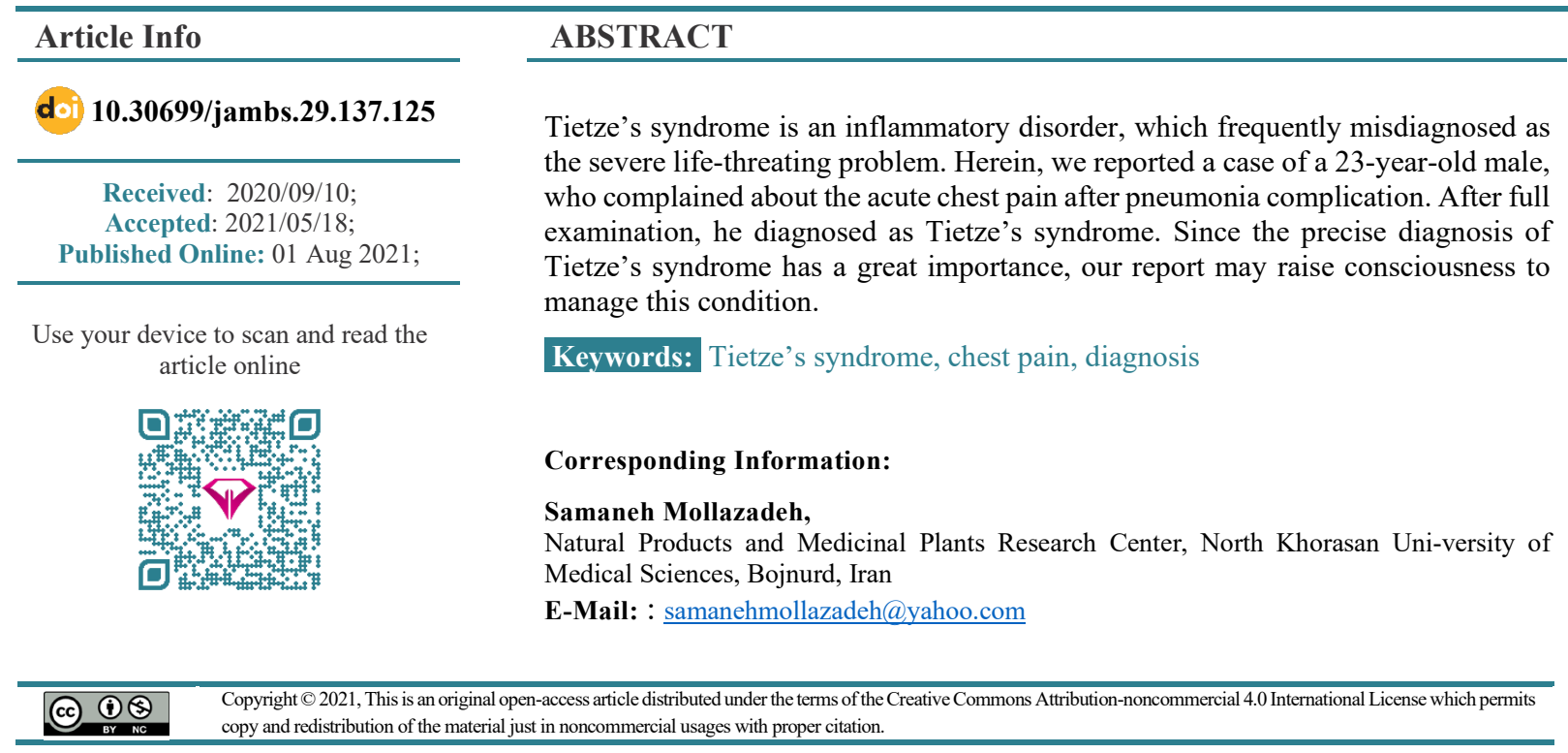

\section{Introduction}

One of the most common reasons for primary care consultation is related to chest pain, which is caused by different complications and affects about $20-40 \%$ of people throughout their life. Clinical investigation has shown that chest pain etiology not only depends on serious cardiovascular diseases, but also musculoskeletal pain [1]. Sometimes patients with benign pathological conditions of chest wall pain, incorrectly are hospitalized in the emergency department due to the serious cardiopulmonary diseases [2]. Various musculoskeletal diagnostic causes of chest pain can be explained by costochondritis, sternalis syndrome, costosternal syndrome, or Tietze's syndrome [1]. Herein, we reported an atypical chest pain in a 23 -year-old male following pneumonia.

\section{Case description}

A 23-year-old man presented to our hospital with complaints of shortness of breath, chest pain, and excessive cough accompanied by localized painful swelling, in anterior chest wall in the left border of sternum for about 5 days. A discoloration (erythema) was observed on the skin of the affected part (Figure 1A). He described the pain like stabbing by a knife, which he had never experienced it. The patient tried to stay motionless or move slowly because of his severe pain.

Two weeks before the onset of the symptoms, the patient has been misdiagnosed with pneumonia and was admitted in another hospital. After discharging from that hospital, his chest wall started to swell and became painful. Besides, he was as a tiling factory laborer, who sometimes carried overweight loads.

On physical examination, the only notable finding was a tender swelling approximately $4 \times 6 \mathrm{~cm}$ in diameter over his left anterior sternum, which was very firm and nonfluctuant. His vital signs were as follows; temperature $36.8^{\circ} \mathrm{C}$, blood pressure $110 / 70 \mathrm{mmHg}$, heart rate 78 beats/min, and respiratory rate $16 / \mathrm{min}$. His basic lab tests (complete blood count, CBC; peripheral blood smear, PBS) were within normal limits; the erythrocyte sedimentation rate (ESR) was $45 \mathrm{~mm} /$ Hour. An electrocardiogram and chest radiograph revealed no abnormallities. Chest CT revealed minimal enlargement in the left anterior chondrosternal joint (Figure 1B). Based on the findings, Tietze's syndrome was concluded.

To rule out other diffuse forms of myofascial chest pain, different hematological tests were carried out. In the following, various pain management strategies were off- 
ered, including nonsteroidal anti-inflammatory drugs (NSAIDs) to relive pain, as well as expectorant to relive cough. A few days later the patient has been discharged in a good condition. During the follow-up by telephone, the patient reported no more problems, and his pain slowly reduced over time. Then, he gave up his work, and no longer used any analgesics.

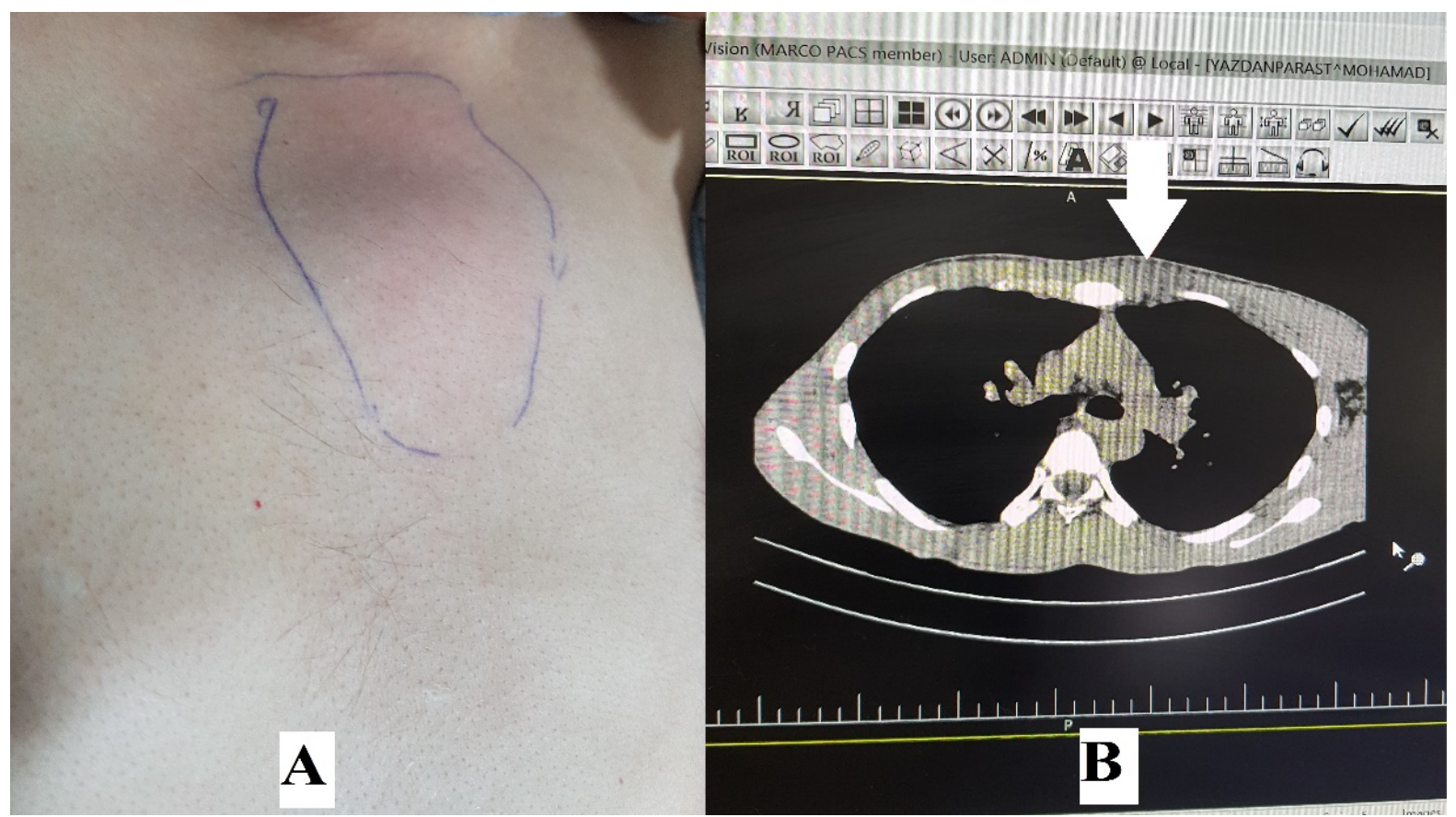

Figure 1. Patient examination results. (A) The patient had swelling over his left anterior sternum joints and tenderness on palpation. (B) Chest computed tomography (CT) demonstrated minimal focal enlargement of the left chondrosternal joint without fracture (white arrow).

\section{Discussion}

Tietze's syndrome has been described by chest pain and swelling of costosternal, costochondral, or sternoclavicular junction, mostly in the second and third ribs [3]. For the first time, it was defined by German professor of surgery Alexander Tietze [4]. This benign syndrome is a relatively rare ailment, most frequently realized [5] in both genders under the age of 40 years [2]. It is thought that the most common reasons of Tietze's syndrome are related to airways infections and/or micro-injuries [6]. To diagnose this complication, physical examination, laboratory tests and imaging studies are applied [3].

Since Tietze's syndrome is a rare disorder, few clinicians have substantial experiences about it. Its mysterious etiology is not dependent on the occupation, geography, or ethnicity [7], however, its diagnostic profile is confused with other thoracic painful conditions [3]. With this regard, physicians should be careful about case history, physical examinations, and test results analysis [8]. Since the syndrome may identify with broad differential diagnosis due to its rarity and location, it is important to rule out life threatening conditions such as gastrointestinal, respiretory and cardiac problems [9].
Also, other probable dangerous issues affecting the chondrosternal joints should be excluded, including tumors, pyogenic and rheumatoid arthritis [8]. Reproducing pain or palpation over the affected area is a pivotal part of clinical examination to confirm musculoskeletal problems. The pain can start suddenly or gradually. Furthermore, bone scintigraphy or CT is not specific enough to distinguish benign costochondral joint disorders from malignant ones [10]. The hallmark of Tietze's syndrome is swelling, which indicates the severity of disorder [9].

In patients above 35 years old complain about painful chest, it is important to exclude coronary heart or cardiorespiratory diseases, using ECG and chest X-ray prior to diagnosis Tietze's syndrome [5]. When the accurate diagnosis is delayed, the emotional and physical stressful conditions will be imposed to the patient [9].

Treatment strategies include pain management using ice and administration of anti-inflammatory mediators and painkillers either topically, orally, or by injection. Another useful therapeutic and diagnostic tool is local injection of anesthesia. In refractory cases, injection of corticosteroid might be deliberated [7]. Here, we presented a young man experiencing acute chest pain consequent of pneumonia with severe cough. The 
diagnostic difficulty in part lies to the absence of convincing laboratory or imaging examinations, which compel us to trust on the patient's history and physical examinations.

This study raises the knowledge about chest wall pain characteristics and the diagnostic accuracy in the emergency department. The acute diagnosis of Tietze's syndrome not only paves the way to choose the best platform for managing the pain, but also save the time and cost to examine other pathological conditions. Most importantly, it provides the emotional and physical relief of patient.

\section{Conclusion}

None.

\section{Authors' contributions}

MRT and MSMH participated in clinical and pathological researches. SM studied related articles and drafted the manuscript. All authors read and approved the final manuscript.

\section{Acknowledgments}

None.

\section{Conflict of Interest}

The authors declare that they have no conflict of interest.

\section{Informed consent}

Informed consent was obtained from the patients included in the study.

\section{Funding}

This research received no specific grant.

\section{References}

1. Bösner S, Becker A, Hani MA, et al. Chest wall syndrome in primary care patients with chest pain: presentation, associated features and diagnosis. Fam Pract. 2010;27(4):363-369. [DOI:10.1093/fampra/cmq024]

2. Sawada K, Ihoriya $H$, Yamada $T$, et al. A patient presenting painful chest wall swelling: Tietze syndrome. World J Emerg Med. 2019;10(2):122-124. [DOI:10.5847/wjem.j.1920-8642.2019.02.011]

3. Rokicki W, Rokicki M, Rydel M. What do we know about Tietze ' s syndrome? Kardiochir Torakochir Pol. 2018;15(3):180-182. [DOI:10.5114/kitp.2018.78443]

4. Tietze A. über eine eigenartige häufung von fällen mit dystrophie der rippenknorpel. Berliner Klin Wochenschrift. 1921;58:829-831.

5. Proulx AM, Zryd TW. Costochondritis: diagnosis and treatment. Am Fam Physician. 2009;80(6):617-620.

6. Jurik AG, Graudal H. Sternocostal joint swelling-clinical Tietze's syndrome. Report of sixteen cases and review of the literature. Scand J Rheumatol. 1988;17(1):33-42. [DOI:10.3109/03009748809098757]

7. Gijsbers E, Knaap SFC. Clinical presentation and chiropractic treatment of Tietze syndrome: A 34year-old female with left-sided chest pain. J Chiropr Med. 2011;10(1):60-63 [DOI:10.1016/i.jcm.2010.10.002]

8. Wertli MM, Ruchti KB, Steurer J, et al. Diagnostic indicators of non-cardiovascular chest pain: a systematic review and meta-analysis. BMC Med. 2013;11(239). [DOI:10.1186/1741-7015-11-239]

9. Grodin L, Farina G. Tietze ' s Syndrome in the emergency department : A rare etiology of atraumatic chest pain. Case Rep Clin Med. 2013;2(3):208-210. [DOI:10.4236/ crcm.2013.23056]

10. Thongngarm $\mathrm{T}$, Lemos $\mathrm{LB}$, Lawhon $\mathrm{N}$, et al. Malignant tumor with chest wall pain mimicking Tietze's syndrome. Clin Rheumatol. 2001;20(4):276278. [DOI:10.1007/PL00011203]

\section{How to Cite This Article:}

Taghavi M R, Mollazadeh S, Mohajerzadeh Heydari M S. Diagnostic challenges in an atypical chest pain, Tietze's syndrome: a case report in Northeast of Iran. J Adv Med Biomed Res. 2021; 29 (137): 359-361

\section{Download citation:}

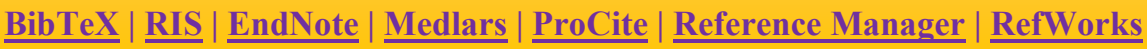

\section{Send citation to:}

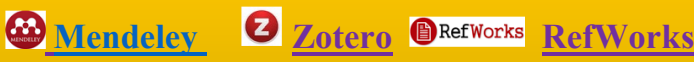

